\title{
Marie-Claire Boscq, Imprimeurs et libraires sous surveillance (1814-1848)
}

Paris, Classiques Garnier, coll. « Littérature et censure », 2018, 464 p., ISBN : 978-2-406-07310-9

Jean-Noël Tardy

\section{OpenEdition} Journals

Édition électronique

URL : https://journals.openedition.org/chs/2968

DOI : $10.4000 /$ chs. 2968

ISSN : 1663-4837

Éditeur

Librairie Droz

\section{Édition imprimée}

Date de publication : 5 août 2021

Pagination : 150-152

ISSN : 1422-0857

Référence électronique

Jean-Noël Tardy, « Marie-Claire Boscq, Imprimeurs et libraires sous surveillance (1814-1848) », Crime, Histoire \& Sociétés / Crime, History \& Societies [En ligne], vol. 25, $n^{\circ} 1$ | 2021, mis en ligne le 05 août 2021 consulté le 10 novembre 2022. URL : http://journals.openedition.org/chs/2968 ; DOI : https://doi.org/ $10.4000 /$ chs. 2968

Ce document a été généré automatiquement le 10 novembre 2022.

Tous droits réservés 


\section{Marie-Claire Boscq, Imprimeurs et libraires sous surveillance (1814-1848)}

Paris, Classiques Garnier, coll. « Littérature et censure », 2018, 464 p., ISBN : 978-2-406-07310-9

Jean-Noël Tardy

\section{RÉFÉRENCE}

Marie-Claire Boscq, Imprimeurs et libraires sous surveillance (1814-1848), Paris, Classiques Garnier, coll. «Littérature et censure », 2018, 464 p., ISBN : 978-2-406-07310-9

1 L'ouvrage, issu d'une thèse de doctorat dirigée par Jean-Yves Mollier, porte sur un aspect peu étudié de l'histoire du livre au XIXe siècle : la mise sous surveillance, à l'initiative de Napoléon, des instruments de la diffusion de l'écrit, les imprimeurs et les libraires. En effet, la Révolution française a provoqué une floraison d'imprimés de natures diverses, tout en désorganisant le monde du livre. La suppression des corporations a entraîné une multiplication de petits imprimeurs, se livrant une concurrence destructrice. Napoléon, par le décret du 5 février 1810, crée une administration spécifique qualifiée de Librairie avec une majuscule, pour réguler mais aussi surveiller le monde du livre, la librairie, cette fois écrite avec une minuscule. Il limite de manière draconienne le nombre d'imprimeurs parisiens à 80 , et cette disposition reste valable pour toute la période étudiée.

2 L'ouvrage porte sur une ville, Paris, la «capitale du livre » et des Révolutions du XIX siècle. Il traite d'une peur politique: celle des monarchies censitaires face à la démocratisation de l'imprimé. Ces régimes, qui prétendent marquer une rupture avec l'autoritarisme impérial, sont obsédés par l'état de l'esprit public, comme l'a démontré Pierre Karila Cohen. Et si la censure de l'imprimé reste difficile à assumer, la surveillance des imprimeurs et des libraires n'est quant à elle jamais véritablement remise en cause. Significativement, le brevet pour les imprimeurs et libraires, dispositif capital de la législation impériale, est maintenu jusqu'en 1870. 
3 L'étude des dispositions réglementaires et leur évolution de 1814 à 1848 suggèrent un renforcement $\mathrm{du}$ contrôle, parfois à rebours du libéralisme affiché par les gouvernements : l'obligation du brevet est étendue en 1817 aux lithographes, en 1826 aux bouquinistes à domicile, aux cabinets de lecture, et même à certains colporteurs. La volonté de contrôle des imprimeurs est plus manifeste encore : la loi du 21 octobre 1814 précise qu'en cas de condamnation, ils perdent leur brevet, ce qui revient à les ruiner. Suite à l'intervention des députés libéraux, la loi du 17 mai 1819 limite les risques de poursuite, si l'imprimeur a rempli les obligations de déclaration et de dépôt. Les tentatives des ultras de durcir cette législation en 1827 puis en 1829 provoquent des réactions virulentes des ouvriers du livre, jusqu'à l'épreuve de force finale et la Révolution des Trois Glorieuses. La procédure d'obtention du brevet prévoit une enquête menée par les services de la Librairie. Il en résulte la constitution d'un fonds d'archives imposant : 300 brevets d'imprimeurs sont distribués entre 1814 et 1848 pour pourvoir et remplacer les 80 propriétaires et 1620 brevets de libraires alors qu'il existe environ 500 librairies en activité sur la période. L'exploitation minutieuse de ces documents est la grande force du livre de Marie-Claire Boscq. En se fondant sur une approche quantitative éprouvée, l'auteur dresse une sociologie de la librairie. Et les rapports d'enquêtes renseignent également sur l'esprit et les techniques de l'institution de la surveillance.

4 L'étude socio-historique du monde des imprimeurs et des libraires précise les travaux des historiens du livre. La géographie des librairies à Paris dépend logiquement de la proximité des universités. Marie-Claire Boscq confirme aussi les effets de l'incendie du Palais Royal en 1827, néfaste pour bien des établissements du quartier. Sociologiquement, les gens du livre sont issus majoritairement de milieux populaires ou de la petite bourgeoisie, en premier lieu de la profession elle-même, libraire ou imprimeur. Les passages consacrés aux femmes détentrices d'un brevet d'imprimeur ( $10 \%$ du total) ou de libraire ( $20 \%$ des effectifs) sont passionnants et inédits. Citant judicieusement les rapports sur les femmes qui sollicitent un brevet ou en disposent, souvent par héritage, Marie-Claire Boscq met en évidence la défiance des autorités envers le travail féminin. La moralité de ces femmes est suspecte, leur compétence jugée douteuse, et seules quelques figures, comme la veuve Panckoucke, voient leur professionnalisme reconnu. L'incongruité de leur faire prêter un serment politique, alors qu'elles ne peuvent être des citoyennes de plein droit, n'échappe à personne.

5 Marie-Claire Boscq consacre ensuite une partie complète aux services chargés de la surveillance de l'écrit à Paris: la Librairie et ses fonctionnaires, mais aussi les commissaires de police et les services de la préfecture de Police. Une double surveillance s'exerce sur le monde du livre, du moins à Paris, car à partir de 1817, les inspecteurs de la Librairie sont supprimés en province. À Paris, à l'exception d'une brève disparition au profit de la police entre 1829 et 1831, les inspecteurs de la Librairie (quatre en moyenne sur la période étudiée) sont maintenus. Ils enquêtent sur les candidats aux brevets, surveillent les ateliers et les boutiques. Les enquêtes ne sont pas des formalités administratives et collectent des informations précises sur les opinions politiques et la moralité des professionnels du livre (ces deux aspects étant inextricablement liés dans l'esprit des gouvernements). Les échanges entre le ministère de l'Intérieur et la préfecture de Police apportent des éclaircissements importants sur les institutions du maintien de l'ordre, notamment sous le ministère Villèle. Ils confirment le peu d'efficacité de la surveillance politique de la police parisienne, avant 
la reprise en main par le préfet Delavau. À ce titre, le moment où les ultras détiennent, sous la direction du ministre de l'Intérieur comte de Corbière, la direction de la police, avec Franchet, et la préfecture de Police, avec Delavau, est un sommet de la répression : 28 librairies sont fermées en novembre 1822 et les demandes de brevet chutent, faute d'espoir d'obtenir le précieux sésame d'une telle administration. La monarchie de Juillet, bien que se réclamant du libéralisme, renoue avec la surveillance des gouvernements précédents. Mais cette politique n'empêche pas une croissance spectaculaire du nombre de livres, de brochures, de pamphlets.

6 À l'évidence et malgré les règles et limitations en vigueur, s'est développée une zone grise : des ateliers d'imprimerie plus ou moins licites existent à Paris ou à proximité de la capitale. Le trafic des imprimés interdits implique parfois les libraires autorisés. L'ouvrage de Marie-Claire Boscq apporte un éclairage précieux sur ces activités interlopes. Pour répondre à la demande croissante en imprimés, les imprimeurs brevetés ont la possibilité d'ouvrir d'autres ateliers qu'ils peuvent confier à un tiers. Certains de ces ateliers sont en fait de véritables éditeurs, indépendants de la maison mère dans leurs choix. On appelle succursalistes ceux qui tiennent ces entreprises abritées par le brevet d'un autre. La Librairie estime leur nombre à 19 dans un rapport de 1833. L'administration accepte même d'accorder des brevets à des succursalistes notoires pour rétablir la légalité. En revanche, échappant à tout contrôle, les imprimeurs marrons s'activent dans la clandestinité. Malgré les efforts de la police qui réprime le trafic du matériel d'imprimerie, ils seraient 28 en 1833.

7 Sur la résistance ouverte ou souterraine de la librairie contre les gouvernements, l'étude de Marie-Claire Boscq donne peu de détails. Il n'est pas simple de restituer la participation des gens du livre aux entreprises de l'opposition politique. L'historienne mentionne la lutte du libraire Ladvocat qui, en 1821, refuse de se plier aux règles et de demander un brevet. Il cède finalement et obtient d'ailleurs le sésame, l'administration craignant que sa fronde ne suscite des imitateurs. Elle cite le nom du libraire Corréard dont le brevet est confisqué, sans faire le lien avec le carbonarisme qui séduit nombre d'éditeurs libéraux. Pour la monarchie de Juillet, on regrettera l'absence de certaines affaires, en particulier celle du Moniteur républicain et de l'Homme libre. Rappelons que le Moniteur républicain, publication clandestine, s'est livré à l'apologie du régicide et l'autorité l'implique dans les tentatives d'assassinat contre Louis Philippe. Les expertises sur les caractères d'imprimerie incriminent un succursaliste fameux: Gambin. En dépit de cette limite, ce livre, solidement documenté, s'impose comme une référence sur la Librairie et le monde du livre parisien, mais informe aussi sur la nature des régimes politiques de 1811 à 1848 . Il confirme l'importance de la période des monarchies censitaires dans le combat pour la démocratisation de l'imprimé en France. 


\section{AUTEURS}

\section{JEAN-NOËL TARDY}

Université Paris-1 Panthéon-Sorbonne

Centre d'histoire du XIX ${ }^{\mathrm{e}}$ siècle

jntardy[at]orange.fr 